\title{
Breast cancer in Western Africa: A pilot molecular analysis of BRCA genes in early- onset breast cancer patients in Burkina Faso
}

Michela Biancolella

Universita degli Studi di Roma Tor Vergata Dipartimento di Biologia

Nabonswindé Lamoussa Marie Ouedraogo

University of Saint Thomas d'Aquin of Ouagadougou

Nayi Zongo

CHU-YO Ougadougou: Centre Hospitalier Universitaire Yalgado Ouedraogo

Théodora Mahoukèdè Zohoncon

University saint thomas d'aquin Ougadougou

Barbara Testa

University of Rome Tor Vergata Department of Biomedicine and Prevention: Universita degli Studi di Roma Tor Vergata Dipartimento di Biomedicina e Prevenzione

\section{Barbara Rizzacasa}

University of Rome Tor Vergata Department of Biomedicine and Prevention: Universita degli Studi di Roma Tor Vergata Dipartimento di Biomedicina e Prevenzione

Andrea Latini

University of Rome Tor Vergata Department of Biomedicine and Prevention: Universita degli Studi di Roma Tor Vergata Dipartimento di Biomedicina e

Prevenzione

Chiara Conte

Azienda Ospedaliera Universitaria Policlinico Tor Vergata: Fondazione PTV Policlinico Tor Vergata

Tégwindé Rebeca Compaore

Pietro Annigoni for Biomolecular Research Centre Ouagadougou

Charlemagne Marie Rayang-Newendé Ouedraogo

Bogodogo University Hospital Center: Centre hospitalier universitaire de Bogodogo

Si Simon Traore

University Saint Thomas d'Aquin, Ougadougou

Jacques Simpore

University Saint Thomas d'Aquin, Ouagadougou

Giuseppe Novelli ( $\nabla$ gnovelli@me.com )

Tor Vergata University of Rome https://orcid.org/0000-0002-7781-602X

Primary research

Keywords: Breast cancer, BRCA1, BRCA2, Burkina Faso, West Africa, NGS

Posted Date: July 15th, 2021

DOI: https://doi.org/10.21203/rs.3.rs-703860/v1

License: @ (1) This work is licensed under a Creative Commons Attribution 4.0 International License. Read Full License 


\section{Abstract}

Background: Breast cancer (BC) is the most commonly diagnosed cancer and the second leading cause of cancer-related deaths, after cervical cancer, among women in Africa. Even if the epidemiological data are now aligned with those relating to industrialized countries, the knowledge concerning breast cancer in Africa, in particularly in Western Africa still lack clinical data, medical treatments, and the evaluation of genetic and non-genetic factors implicated in the etiology of the disease.

The early onset and the aggressiveness of diagnosed breast cancers in patients of African ancestry strongly suggest that the genetic risk factor may play an important role but up to date very few studies have been done concerning the impact of germ line mutations in breast cancer in Africa, with a negative impact on prevention, awareness and patient management.

We have performed by Next Generation sequencing (NGS) the analysis of all coding regions and the exon-intron junctions of $B R C A 1$ and $B R C A 2$ genes, the two most important genes in hereditary breast cancer, in fifty-one women from Burkina Faso with early onset of breast cancer and or without a family history.

Results: We identified six different pathogenic mutations (3 in BRCA1, 3 in BRCA2), two of which have been found to be recurrent, in 8 unrelated women.

In addition, we identified, in 4 other patients, two variants of uncertain clinical significance (VUS) and two variants never previously described in literature, although one of which is present in the dbSNP database.

Conclusions: The present study is the first in which the entire coding sequence of $B R C A$ genes have been analyzed by Next Generation Sequencing in Burkinabe young women with breast cancer.

Our data support the importance of genetic risk factors in the etiology of breast cancer in this population and suggests the necessity to improve the genetic cancer risk assessment. Furthermore, the identification of the most frequent mutations of $B R C A 1$ and $B R C A 2$ in the population of Burkina Faso will allow the development of an inexpensive genetic test for the identification of subjects at high genetic cancer risk, which could be used to design personalized therapeutic protocols.

\section{Background}

The global rate of new cancer cases is increasing worldwide [1]. In 2018, the global cancer observatory estimated that, by 2030,24 million people worldwide will develop cancer and 13 million people will die annually from cancer, with 75\% of these deaths in low-and middle-income countries [2, 3]. In these countries, the global trend in the epidemiology of breast cancer merges what observed for high-income countries [4]. Focusing on Africa and specifically on the area of Western Africa, which comprises Burkina Faso, breast cancer represents the second most frequent diagnosed cancer in women [4,5]. However, the lack of resources for preventive screening and access to quality healthcare lead to significant delays in breast cancer detection contributing to a high mortality rate in these countries [6, 7]. Moreover, the genetics of breast cancer in African countries is generally uncertain [8] and also if the early age of onset and the aggressiveness suggest that there may be a strong inheritance-familial component in the onset of the disease, very few studies have attempted to fill this lack of acknowledge $[8,9,10,11]$.

In order to better assess the genetic cancer risk in this population we analyzed by next generation sequencing (NGS) the coding regions and the exon-intron junctions of the two main breast cancer susceptibility genes, [12], BRCA1 [13] and BRCA2 [14], in 51 women from Burkina Faso affected by breast cancer.

To the best of our knowledge, this is the first work that analyzed, with an NGS approach, all the coding sequence of $B R C A 1$ and $B R C A 2$ genes in Burkinabe women with breast cancer. The identification and characterization of the most recurrent mutations in these patients will allow the development of genetic tests based on a population-specific mutations panel thus decreasing the cost of genetic testing. Therefore there will be the possibility of carrying out preventive screenings in high-risk populations.

Moreover, the early identification of women carrying germline pathogenic or likely pathogenic variants in $B R C A 1$ or $B R C A 2$ genes will allow to set up an appropriate diagnostic-therapeutic path to improve the overall survival rate of breast cancer patients.

\section{Results}

A total of 51African women from Burkina Faso affected by breast cancer have been selected by genetic counseling at CERBA/LABIOGENE laboratory of University of Ouagadougou (Burkina Faso) to perform a genetic testing for the screening of BRCA1 and BRCA2 genes. The molecular analyses were carried out at the Medical Genetics Laboratory of University of Rome Tor Vergata.

All patients had an age at diagnosis less than 40 years. The mean age of patients at diagnosis was $34.8 \pm 4.14$. Ninety-four percent ( $94 \%)$ of patients had an invasive ductal type of breast carcinoma, and only $24 \%(12 / 51)$ reported to have family history of breast cancer.

The NGS analysis produced an output of 644 entries containing the variant code, chromosomal, coding and amino acidic position. Only 30 of them have been found just one time in a single patient. Of these 30 unique variants (exonic and intronic), 4 are pathogenic (13\%), 2 are variants of uncertain significance (VUS) (7\%), 2 are novel (7\%) and 22 are benign variants (73\%).

Overall, on the total of 51 patients analyzed we found 8 carriers of a pathogenic variant (16\%), 2 carriers of a variant of uncertain significance (VUS) (4\%) and 2 carriers of a new undescribed variant (4\%) (Fig. 1). We observed that the median age of patients carrying pathogenic variants was lower (33.25 \pm 3.77 ) compared to the median age of patients carrying only benign variants $(35.16 \pm 4.16)$, although this difference was not statistically significant $(p$ value $=0.25)$. 
The identified pathogenetic variants were in total 6 ( 4 unique and 2 recurrent in more than one patient). Three variants were in $B R C A 1 \mathrm{gene}$ and 3 in $B R C A 2$ gene. The $B R C A 1$ mutation carriers had a mean age of (32.4 \pm 3.78$)$ years, $B R C A 2$ mutations carriers had a mean age of (34.67 \pm 4.04$)$. The mutation prevalence was evaluated in our cohort and resulted to be $15.7 \%$ (95\% Cl: $5.7-25.7 \%$ ) for the two $B R C A$ genes, in particular $9.8 \%$ (95\% $\mathrm{Cl}$ : $1.6-18.0 \%$ ) for BRCA1 and 5.9\% (95\% Cl: $0.2-11.4 \%)$ for BRCA2. The mutations included missense, nonsense, small deletion and intronic variants (Table 1). In particular, in $B R C A 1$ gene we identified the presence of 2 recurrent pathogenic variants in 4 unrelated patients. Specifically, 2 patients were affected by the same mutation in $B R C A 1$ gene, c. $4088 \mathrm{C}>\mathrm{G}$, (p.Ser1363*). This is a nonsense mutation which causes the substitution at amino acid 1363 from Serin to a stop codon. Patients carrying this mutation had an undifferentiated and ductal type of breast carcinoma at ages of 37 and 34 years, respectively, and both had a family history of breast cancer. Other 2 patients shared the intronic mutation c.4986 +6T >C ( LRG_292t1:c.4986 +6T >C) in BRCA1 gene. This variant has a severe impact on splicing because leads to activation of a downstream cryptic splice donor site, which results in an aberrant RNA transcript and a truncated protein. These patients were affected by a breast ductal carcinoma, diagnosed at early ages of 28 and 29 years, respectively. One patient reported to have family history of cancer, while the other patient did not. Moreover we identified 2 frameshift mutations in BRCA2 gene, the c.6445_6446del, (p.lle2149*) and the c.6757_6758del, (p.Leu2253Phefs*7) and 1 frameshift mutation in BRCA1 gene, c.5177_5180del,(p.Arg1726Lysfs*3) .Two of the frameshift variants identified were in women with no family history of breast cancer. The only missense mutation identified, c.8009C > T, (p. Ser2670Leu), was in BRCA2 gene. The variant was present in a 37-years-old woman with a medullary breast carcinoma and a family history for breast cancer. Interestingly, the pathogenetic variants frequency detected in our cohort is statistically different respect to the frequencies listed in the GnomAD database for the African population ( $p$-value < 0.05 ), with the two variants: the c.6445_6446del, (p.lle2149*) and the c.8009C > T, (p.Ser2670Leu), which are not even present in GnomAD database (Table 1). In addition to the pathogenetic alleles we identified two missense variants of uncertain significance (VUS); one in BRCA1 gene (c.5348T >C, p.Met1783Thr) and one in BRCA2 gene (c.7504C > T, p.Arg2502Cys) with an allelic frequency not statistically different respect to the frequencies listed in the GnomAD database for the African population ( $p$-value $>0.05$ ) (Table 1). Both these variants are in a functional domain of the BRCA1 and BRCA2 protein, respectively. The variant Met1783Thr is in the BRCT2 domain of BRCA1, while the variant Arg2502Cys is in the BRCA2 helical domain. To predict the potential impact of these variants on the protein we used different tools (Mutation Taster and PolyPhen-22). The in silico analysis predicted a damaging role for the BRCA1 variant (Mutation Taster: disease causing; PolyPhen-2: Probably damaging, with a score of 1.000); moreover the sequence alignment of the BRCA1 protein with its orthologous proteins showed that the wild type residue seemed to be moderately preserved in species, implying a role for this residue in the protein function. The in silico analyses for the BRCA2 variant Arg2502Cys gave a benign computational effect on the protein (Mutation Taster: polymorphism; PolyPhen-2: benign, with a score of 0.022 ). In this case the sequence alignment of BRCA2 protein with its orthologous proteins showed that the wild type residue is poorly conserved among species implying an irrelevant functional or structural role of this residue on the protein.

Finally, we have identified 2 unclassified variants (4\%) in BRCA1 gene: 1 missense variant, c.2359G >A, (p.Glu787Lys) (rs1288796003) and 1 nonsense variant, c.872T > A, (p.Leu291*). We used in silico tools (Mutation Taster and PolyPhen-22) to evaluate the potential impact of these variants on BRCA1 protein.

The missense variant c. $2359 \mathrm{G}>\mathrm{A}$ causes a non-conservative amino acid change, the substitution of Glutamic Acid 787 to Lysine. This variant is present on dbSNP database (rs1288796003) but is not present on any clinical databases (ClinVar, LOVD) and is not reported on GnomAD. The in silico analysis with different software gave conflicting verdicts.

The variant c.872T > A, (p.Leu291*) cause the changing of amino acid 291 from Leucine to stop codon thus breeding a truncated protein that comes shorter of 6916 amino acids. In accordance with the American College of Medical Genetics (ACMG) this variant is classified likely-pathogenic-class 4 [15].

\section{Discussion}

In Africa and Sub-Saharan Africa (SSA) breast cancer (BC) is the most diagnosed cancer in women with an increasing incidence in the last few years, and a five-year survival rate lower compared to industrialized countries [16]. Epidemiological studies would be very useful to better identify risk factors and understand the genetic variability among African populations. However, up to date, few data are available, with a significant impact on the accuracy of diagnosis and clinical management of patients with African ancestry.

The prevalence of mutations in BRCA genes is not yet well defined in Western Africa. Data are scarce for most countries and the results of the few studies carried out do not allow to have a real picture of the specific mutations in each country. The prevalence and spectrum of germline mutations in $B R C A 1$ and $B R C A 2$ genes are certainly better delineated in European and North American populations [17]. For many of these populations recurrent and founder mutations have been identified and this has allowed the development of targeted genetic tests which, being less expensive, can be more easily used for population screening [18]. One example is represented by the Ashkenazi Jewish population in which identified founder mutations have long been used as the first genetic screening test for women of Jewish descent [19]. Founder mutations have also been identified in different European and Asian populations, while for West Africa only one mutation in BRCA1 gene has been identified as a potential founder mutation [20]. These examples suggest that specific mutation panels can be developed for specific populations. Therefore it is important for Africa identify and characterize the recurrent mutations [11].

In this study, we determined the prevalence of mutations in BRCA genes in a cohort of young Burkinabe women with breast cancer. Interestingly, the frequency of all identified pathogenic variants, some of which are present in more than one patient, was statistically different from that one reported in the GnomAD database for the African population, with two variants that are not even present in the database. This result suggests that the identified variants could be considered as population specific variants and therefore be extremely important for genetic testing strategies. In addition to the pathogenetic variants, we have identified 2 variants of uncertain clinical significance (VUS) and 2 variants never described in the literature of which only one has been previously reported only in the dbSNP database. The two VUS are in functional domains, and the in silico analysis have predicted a damaging role for the one in $B R C A 1$ gene and a benign computational effect on the protein for the one in BRCA2 gene. Both variants have an allelic frequency not statistically different to the one listed in the GnomAD database for the African population suggesting their unlikely contribution to disease risk on their own. However, the role of these variants 
should certainly be better investigated using multifactorial models and functional studies [21]. In this regard to overcome difficulties in classifying $B R C A 1 / 2$ VUS, in 2009 was born the Evidence-Based Network for the Interpretation of Germline Mutant Alleles (ENIGMA) consortium [22]. The International Agency for Research on Cancer Working Group (IARC), in collaboration with ENIGMA, has developed a five-level multifactorial model to classify VUS identified in BRCA genes, based on the segregation of the variant in families, the co-occurrence with previously identified pathogenic mutations and tumor histopathology, combined with an analysis of the sequence conservation and properties of mutated residues [23].

In our study, we could not carry out the segregation analysis as we did not have the DNA of others family members nor functional studies, the only analysis that have been performed were those using the in silico tools.

The two novel variants identified were in the BRCA1 gene. One is a nonsense variant that, in accordance with ACMG and ENIGMA criteria, we have classified as likely-pathogenic, the other is a missense variant that needs further investigations. Finally, 17 patients (those with better quality DNA) tested negative for BRCA1/2 mutations were screened for larger genomic rearrangements (LGRs) in BRCA genes by multiplex ligation-molecular dependent probe amplification (MLPA). No genomic rearrangements were identified in any of the patients analyzed. This data is in agreement with the results obtained by Zhang j et al [24], showing that the genomic rearrangements do not contribute significantly to BRCA-associated risk in the Nigerian population.

Our study has limitations. One is certainly represented by the low number of patients analyzed, another is that the genes screened were only the $B R C A$ genes. Up to date, the majority of African BC genetic studies concern only the molecular analysis of $B R C A$ genes which leads to an inaccurate characterization of $B C$ genetic risk factors. Our next step will be to analyze molecular test negative patients for $B R C A 1 / 2$ mutations with a larger panel that includes other important breast cancer susceptibility genes in order to have a more complete picture of genetic risk factors in Africa such as in European and American countries [25, 26].

\section{Conclusions}

Certainly the socio-economic conditions that lead to a weak healthcare system, the lack of health insurance, limited access to drugs and therapies and a lack of genetic tests have a strong impact to the high mortality and incidence rates of breast cancer in the African countries. We believe that our study, although conducted on a limited number of patients, represents an important contribution to add greater knowledge to the genetic risk factors of BC in Western Africa. This will permit to have cancer prevention programs and will allow Burkinabe women at high risk of breast cancer to be included in an appropriate diagnostictherapeutic programs. In fact, it will help reduce the disparities that still exist between being a breast cancer patient in a low-income or a high-income country.

\section{Methods}

\section{Patients' recruitment}

This was a prospective cohort study which took place from August Ist, 2015 to February 29th, 2016. It consisted of a genetic analysis of breast cancer confirmed cases by histopathological analyses among women younger than 40 years at the University hospital Center of Yalgado OUEDRAOGO (CHU-YO). An approval was obtained from the Ethics Committee for Health Research of Burkina Faso ( $N^{\circ}$ 2014-8-098). After obtaining written informed consent from each patient, clinical, paraclinical and therapeutic data were collected in General Surgery, Gynecology-Obstetrics, Oncology and Anatomy-Pathology departments of the CHU-YO. Women younger than 40 years with histologically confirmed breast cancer, attending the latter mentioned depatments, who gave their free and written consent to participate, were included in the present study.

The parameters studied for each patient were:

Epidemiological and socio-demographic characteristics (age, sex, occupation, weight, height, level of education, origin); clinical data (antecedents, consultation time, reasons for consultation, symptoms and physical signs).

Data collection was possible through interview, physical exanimation, and investigations. For each patient, the tumor was classified according to the cTNM (clinical classification in Tumor-Nodes-Metastasis) and pTNM ( $p$ = pathologic) classification of breast cancers (7" edition 2010), and graded according to Scarff-Bloom and Richardson (SBR) grading system.

\section{DNA extraction and NGS analysis}

DNA extraction and NGS analyses were performed at Medical Genetics Laboratory of University of Rome Tor Vergata.

Total DNA was isolated from peripheral blood using the QIAGEN® EZ1 DNA Blood $200 \mu$ l kit (Qiagen) with the BioRobot EZ1 Workstation (Qiagen, Valencia, CA, USA). The concentration and quality of DNA was determined using NanoDrop 1000 (Thermo Fisher Scientific) and the Qubit Fluorometer 2.0 (Thermo Fisher Scientific).

The NGS analyses were performed using lon AmpliSeq ${ }^{\text {TM }}$ BRCA1 and BRCA2 custom Panel (Thermo Fisher Scientific, Inc). The panel consists of three primers pools ( 55 amplicons) targeting the entire coding region and the exon-intron boundaries of genes BRCA1 and BRCA2. A total of 10 ng of DNA for sample was used for library preparation, using the Ion AmpliSeq ${ }^{\text {TM }}$ Library kit 2.0 (Ion Torrent; Thermo Fisher Scientific, Inc.). Each library was barcoded using lon Xpress ${ }^{\mathrm{TM}}$ Barcode Adapters kit (Ion Torrent; Thermo Fisher Scientific, Inc.). After the amplification phase follows the emulsion reaction that creates aqueous droplets that randomly trapped one or more DNA fragments. Libraries were purified using Agencourt Ampure XP Beads, quantified with the Qubit version 2.0 fluorometer (Thermo Fisher Scientific) using the Qubit dsDNA HS assay kit and diluted approximately 100 pmol/L for PGM while for S5 30 pM. Templated lon Sphere Particles (ISPS) were loaded into an Ion 510 Chip (Thermo Fisher Scientific) or lon 316 Chip (Thermo Fisher Scientific). Sequencing was performed on 
an lon S5 Platform using the lon S5 Sequencing kit (Thermo Fisher Scientific) and on lon PGM Platform using the lon PGM sequencing kit. All protocols were followed as recommended by the manufacturers without modification.

\section{Data analysis}

Preinstalled plugin in the Torrent Browser generates Binary Alignment/Map (BAM) and variant call format (VCF) files. Raw sequence data were processed using the Torrent Suite ${ }^{T w}$ software (Ion Torrent; Thermo Fisher Scientific, Inc.) to analyze barcode reads, to align reads to the HG19 reference genome (Genome Reference Consortium GRCh37) and to generate run metrics, including chip loading efficiency and total read counts and quality. Coverage analysis and variant calling used Torrent Variant Caller plugin software in the Torrent Server. We analyzed bam files on IGV (Integrative GenomeViewer) [27] to verify the real coverage of genes and the presence of variants, and on Ion Reporter Thermo Fisher Scientific, Inc.) that allow annotation of single nucleotide variants, insertions, deletions and splice site alterations.

Variants were annotated according to nomenclature used by the Human Variation Society [28]. All the annotations and variants were determined using BRCA1 (NM_007294.3) and BRCA2 (NM_000059.3) as reference transcripts. All candidate variants were required on both sequenced DNA strands with a minimum depth of $50 \mathrm{X}$.

\section{Variant classification}

All the detected sequence variations were submitted to following databases: BRCA Exchange [29], ClinVar database [30], Leiden Open Variation Database (LOVD) [31], and compared with literature data.

The in silico analysis to predict the potential impact of the variants on the structure and function of the protein was performed using the following tools: PolyPhen2 [32], and Mutation Taster [33].

The evaluation of the novel variants has been based on: the location, type and evolutionary conservation of mutated amino acids, biophysical and biochemical differences between wild-type and mutant amino acid, the in silico analysis of the mutant sequence protein.

\section{Results' validation by Sanger sequencing}

All the variants of class 3 (variants uncertain significance) class 4/5 (likely pathogenic/pathogenic) and the novel variants detected by NGS were confirmed by bidirectional Sanger sequencing.

The sequencing was performed using the BigDye Terminator v3.1 Cycle Sequencing kit (Applied Biosystems; Thermo Fisher Scientific, Inc.) and the ABI $3130 x I$ Automated Sequencer (Applied Biosystems, Foster City, CA, USA). The results were analyzed using Sequencing Analysis 5.2.0 software.

\section{Multiplex Ligation -dependent PCR Amplification (MLPA)}

The presence of large genomic rearrangements (LGRs) in BRCA1 and BRCA2 gene, in addition to the presence of the 1100delC mutation in the CHEK2 gene, was investigated by multiplex ligation-dependent probe amplification (MLPA) assay using MLPA commercial kits from MRC Holland (Multiplex Ligationdependent Probe Amplification, BRCA1: P002, BRCA2: P045) according to the manufacturer's instructions. We used $100 \mathrm{ng}$ of DNA from each sample, three reference samples and tests were performed in duplicate in the same experiment.The procedures were performed according to the manufacturer's instructions.. The analysis of fragments was performed on ABI 3130xl sequencer and the data generated were imported and analyzed in Coffalyser.Net Software (v.140721.1958).

\section{Statistical Analysis}

In this study, the analyses focused only on mutations that are classified as pathogenic. We calculated the mutation prevalence and exact $95 \%$ confidence interval $(\mathrm{Cl})$ using a Binomial distribution. Differences between alleles frequencies of our examined cohort and those listed in the GnomAD database [34] for the African population were evaluated by Fisher's exact test. $p$-values less than 0.05 were considered statistically significant.

\section{Declarations}

\section{Ethics approval and consent to participate}

This work has been approved by the Ethical Committee for Health Research of Burkina Faso $\mathrm{N}^{\circ}$ 2014-8-098. Each subject enrolled in the study signed a written informed consent. The research related to human use has been complied with all the relevant national regulations, institutional policies and in accordance the tenets of the Helsinki Declaration.

Availability of data and materials

All data generated or analysed during this study are included in this published article.

\section{Competing interests}

The authors declare that they have no competing interests.

\section{Funding}

This research has been financed by the Pietro Annigoni Association of Italy 
Design of experiments: ONLM, ZN, TMZ, CTR, SJ. Performance of the experiments: ONLM, BM, ZN, TB, CC, CTR, TMZ, SJ. Data analysis: BM, ONLM, TB, RB, LA, SJ, NG. Contribution in terms of reagents/materials/analysis tools: BM, CC, CTR, SJ. Paper drafting: BM, ONLM, TB, RB, LA, OCM, ZN, TSS, SJ, NG. The author (s) read and approved the final manuscript.

Acknowledgements

We would like to thank all the patients who agreed to participate in this study; then, the departments of general surgery, obstetrics and gynecology, oncology and anatomy pathology of the University Hospital Yalgado Ouédraogo; Pietro Annigoni Biomolecular Research Center (CERBA),

\section{References}

1. Bray F, Ferlay J, Soerjomataram I, Siegel RL, Torre AL, Jemal A. Global cancer statistics 2018: GLOBOCAN estimates of incidence and mortality worldwide for 36 cancers in 185 countries. CA Cancer J Clin. 2018. doi:10.3322/caac.21492.

2. The Lancet. GLOBOCAN 2018: counting the toll of cancer. Lancet. 2018. doi.org/10.1016/S0140-6736(18)32252-9.

3. Ferlay J, Ervik M, Lam F, et al. Global Cancer Observatory: Cancer Today (2018). In: Lyon, editor, France: International Agency for Research on Cancer [https://gco.iarc.fr/today].

4. International Agency for Research on Cancer. Cancer in Africa: epidemiology and prevention. IARC Sci Publ. 2003;153:1-414.

5. Parkin DM, Bray F, Ferlay J, Jemal A. Cancer in Africa 2012. Cancer Epidemiol Biomarkers Prev. 2014;23:953-66. doi:10.1158/1055-9965.EPI-14-0281.

6. Black E, Richmond R. Improving early detection of breast cancer in sub-Saharan Africa: why mammography may not be the way forward. Global Health. 2019;15:3-4.

7. Cumber SN, Nchanji KN, Tsoka-Gwegweni JM. Breast cancer among women in sub-Saharan Africa: prevalence and a situational analysis. Southern African Journal of Gynaecological Oncology. 2017;9:35-7.

8. Sagna T, Bonora E, Ouedraogo MNL, Fusco D, Zoure AA, Bisseye C, et al. Identification of BRCA1/2 p.Ser1613Gly, p.Pro871Leu, p.Lys1183Arg, p.Glu1038Gly, p.Ser1140Gly, p.Ala2466Val, p.His2440Arg variants in women under 45 years old with breast nodules suspected of having breast cancer in Burkina Faso. Biomol Concepts. 2019;10:120-7. doi:10.1515/bmc-2019-0015.

9. Zouré AA, Bambara HA, Sawadogo AY, Bakri Y, Simporé J. BRCA1 and BRCA2 gene mutations in breast cancer among west African women. African J Biomedical Res. 2018;21:7-10.

10. Abbad A, Baba H, Dehbi H, Elmessaoudi-Idrissi M, Elyazghi Z, Abidi O, et al. Genetics of breast cancer in African populations: a literature review. Glob Health Epidemiol Genom. 2018. doi:10.1017/gheg.2018.8.

11. Zoure AA, Slaoui M, Bambara HA, Sawadogo AY, Compaoré TR, Ouédraogo NLM, et al. BRCA1 c.68_69delAG (exon2), c.181T > G (exon5), c.798_799delTT and 943ins10 (exon11) mutations in Burkina Faso. J Public Health Afr. 2018;9:663. doi:10.4081/jphia.2018.663.

12. Petrucelli N, Daly MB, Pal T. BRCA1- and BRCA2-Associated Hereditary Breast and Ovarian Cancer. In: Adam MP, Ardinger HH, Pagon RA, Wallace SE, Bean LJH, Mirzaa G, Amemiyae A, editors. GeneReviews. Seattle (WA): University of Washington, Seattle; 1993-2021.

13. Miki Y, Swensen J, Shattuck-Eidens D, Futreal PA, Harshman K, Tavtigian S, et al. A strong candidate for the breast and ovarian cancer susceptibility gene BRCA1. Science. 1994;266:66-71. doi:10.1126/science.7545954.

14. Wooster R, Bignell G, Lancaster J, Swift S, Seal S, Mangion J, et al. Identification of the breast cancer susceptibility gene BRCA2. Nature. 1995;378:78992. doi:10.1038/378789a0.

15. Richards S, Aziz N, Bale S, Bick D, Das S, Gastier-Foster J, et al. Standards and guidelines for the interpretation of sequence variants: A joint consensus recommendation of the American College of Medical Genetics and Genomics and the Association for Molecular Pathology. Genet Med. 2015;17:405-24. doi:10.1038/gim.2015.30.

16. Ssentongo P, Lewcun JA, Candela X, Ssentongo AE, Kwon EG, Ba DM, et al. Regional, racial, gender, and tumor biology disparities in breast cancer survival rates in Africa: a systematic review and meta-analysis. PLoS One. 2019;14:e0225039. doi:10.1371/journal.pone.0225039.

17. Rebbeck TR, Friebel TM, Friedman E, Hamann U, Huo D, Kwong A, et al. Mutational spectrum in a worldwide study of 29,700 families with BRCA1 or BRCA2 mutations. Hum Mutat. 2018;39:593-620. doi:10.1002/humu.23406.

18. Nanda R, Schumm LP, Cummings S, Fackenthal JD, Seven L, Ademuyiwa F, et al. Genetic testing in an ethnically diverse cohort of high-risk women: a comparative analysis of BRCA1 and BRCA2 mutations in American families of European and African ancestry. JAMA. 2005;294:1925-33. doi:10.1001/jama.294.15.1925.

19. Rebbeck TR, Mitra N, Wan F, Sinilnikova OM, Healey S, McGuffog L, et al. Association of Type and Location of BRCA1 and BRCA2 Mutations with Risk of Breast and Ovarian Cancer. JAMA. 2015;313:1347-61. doi:10.1001/jama.2014.5985.

20. Mefford HC, Baumbach L, Panguluri RC, Whitfield-Broome C, Szabo C, Smith S, et al. Evidence for a BRCA1 founder mutation in families of West African ancestry. Am J Hum Genet. 1999;65:575-8. doi:10.1086/302511.

21. Toland AE, Andreassen PR. DNA repair-related functional assays for the classification of BRCA1 and BRCA2 variants: a critical review and needs assessment. J Med Genet. 2017;54:721-31. doi:10.1136/jmedgenet-2017-104707.

22. Spurdle AB, Healey S, Devereau A, Hogervorst FB, Monteiro AN, Nathanson KL, et al. ENIGMA-evidence-based network for the interpretation of germline mutant alleles: an international initiative to evaluate risk and clinical significance associated with sequence variation in BRCA1 and BRCA2 genes. Hum Mutat. 2012;33:2-7. doi:10.1002/humu.21628. 
23. Plon SE, Eccles DM, Easton D, Foulkes WD, Genuardi M, Greenblatt MS, et al. Sequence variant classification and reporting: recommendations for improving the interpretation of cancer susceptibility genetic test results. Hum Mutat. 2008;29:1282-91. doi:10.1002/humu.20880.

24. Zhang J, Fackenthal JD, Huo D, Zheng Y, Olopade Ol. Searching for large genomic rearrangements of the BRCA1 gene in a Nigerian population. Breast Cancer Res Treat. 2010;124:573-7. doi:10.1007/s10549-010-1006-9.

25. Tsaousis GN, Papadopoulou E, Apessos A, Agiannitopoulos K, Pepe G, Kampouri S, et al. Analysis of hereditary cancer syndromes by using a panel of genes: novel and multiple pathogenic mutations. BMC Cancer. 2019;19:535. doi:10.1186/s12885-019-5756-4.

26. Breast Cancer Association Consortium. Dorling L, Carvalho S, Allen J, González-Neira A, Luccarini C, et al. Breast Cancer Risk Genes-Association Analysis in More than 113,000 Women. N Engl J Med. 2021;384:428-39. doi:10.1056/NEJMoa1913948.

27. The Broad Institute. and the Regents of the University of California. .

28. The Human Genome Variation Society. http://www.hgvs.org.

29. The BRCA, Exchange. https://brcaexchange.org/.

30. Landrum MJ, Lee JM, Benson M, Brown GR, Chao C, Chitipiralla S, et al. ClinVar: improving access to variant interpretations and supporting evidence. Nucleic Acids Res. 2018;46: D1062-7. doi:10.1093/nar/gkx1153. http://www.ncbi.nlm.nih.gov/clinvar/.

31. Fokkema IF, Taschner PE, Schaafsma GC, Celli J, Laros JF, den Dunnen JT. LOVD v.2.0: the next generation in gene variant databases. Hum Mutat. 2011;32:557-63. https://www.lovd.nl/.

32. Adzhubei IA, Schmidt S, Peshkin L, Ramensky VE, Gerasimova A, Bork P, et al. A method and server for predicting damaging missense mutations. Nat Methods. 2010;7:248-9. http://genetics.bwh.harvard.edu/pph2/.

33. Schwarz JM, Cooper DN, Schuelke M, Seelow D. MutationTaster2: mutation prediction for the deep-sequencing age. Nat Methods. 2014;11:361-2. http://www.mutationtaster.org/.

34. Karczewski KJ, Francioli LC, Tiao G, Cummings BB, Alföldi J, Wang Q, et al. The mutational constraint spectrum quantified from variation in 141,456 humans. Nature. 2020;581434-43. doi.org/10.1038/s41586-020-2308-7. https://gnomad.broadinstitute.org.

\section{Tables}

Table 1. Pathogenic variants and VUS identified in our study.

\begin{tabular}{|c|c|c|c|c|c|c|c|c|c|c|}
\hline & Gene & Location & $\begin{array}{l}\text { HGSV } \\
\text { nucleotide }\end{array}$ & HGSV protein & $\begin{array}{l}\text { Number } \\
\text { of } \\
\text { carriers }\end{array}$ & dbSNP & $\begin{array}{l}\text { Allele } \\
\text { Frequency }\end{array}$ & $\begin{array}{l}\text { Allele } \\
\text { Frequency } \\
\text { in Africa } \\
\text { (gnomAD) }\end{array}$ & p-value & $\begin{array}{l}\text { All } \\
\text { populatiol } \\
\text { Frequency }\end{array}$ \\
\hline \multirow{6}{*}{$\begin{array}{l}\text { Pathogenic } \\
\text { Variants }\end{array}$} & \multirow[t]{3}{*}{$B R C A 1$} & Exonic & c.5177_5180del & p.Arg1726Lysfs*3 & 1 & rs80357975 & $0.98 \%$ & $0.012 \%$ & 0.01 & $0.0008 \%$ \\
\hline & & Exonic & c. $4088 \mathrm{C}>\mathrm{G}$ & p.Ser1363* & 2 & rs398122680 & $1.96 \%$ & $0.006 \%$ & 0.00003 & $0.0004 \%$ \\
\hline & & Intronic & c. $4986+6 \mathrm{~T}>\mathrm{C}$ & p.(?) & 2 & rs80358086 & $1.96 \%$ & $0 \%$ & 0.00001 & $0.0004 \%$ \\
\hline & \multirow[t]{3}{*}{ BRCA2 } & Exonic & c.6445_6446del & p.lle2149* & 1 & rs80359592 & $0.98 \%$ & $\begin{array}{l}\text { Not } \\
\text { Reported }\end{array}$ & - & - \\
\hline & & Exonic & $\mathrm{c} .8009 \mathrm{C}>\mathrm{T}$ & p.Ser2670Leu & 1 & rs80359035 & $0.98 \%$ & $\begin{array}{l}\text { Not } \\
\text { Reported }\end{array}$ & - & - \\
\hline & & Exonic & c.6757_6758del & p.Leu2253Phefs*7 & 1 & rs80359623 & $0.98 \%$ & $0 \%$ & 0.003 & $0.0004 \%$ \\
\hline \multirow[t]{2}{*}{ VUS } & $B R C A 1$ & Exonic & c. $5348 \mathrm{~T}>\mathrm{C}$ & p.Met1783Thr & 1 & rs55808233 & $0.98 \%$ & $0.17 \%$ & 0.16 & $0.016 \%$ \\
\hline & $B R C A 2$ & Exonic & c.7504C>T & p.Arg2502Cys & 1 & rs55716624 & $0.98 \%$ & $0.32 \%$ & 0.28 & $0.033 \%$ \\
\hline
\end{tabular}

Significant differences are reported in bold.

\section{Figures}




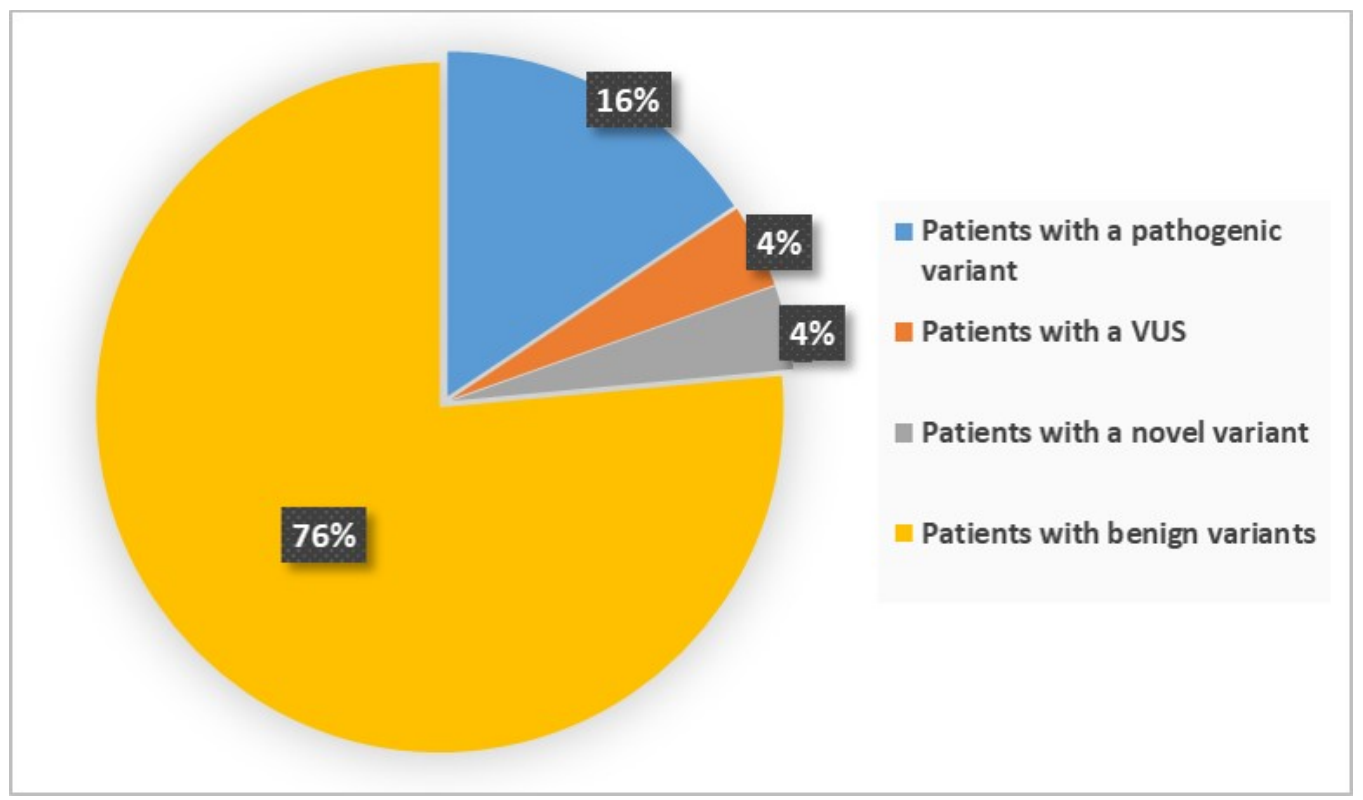

Figure 1

Percentage of patients carrying a pathogenic variant (16\%), a VUS (4\%), a novel variant (4\%) or benign variants $(76 \%)$. 